\title{
Structural and Optical Characterization of Biosynthesized CdS Quantum Dots
}

\author{
Zhou Yang ${ }^{1}, \underline{\mathrm{Li} \mathrm{Lu}}^{2}$, Victoria F. Berard ${ }^{1}$, Christopher J. Kiely ${ }^{2}$, Steven McIntosh ${ }^{1}$ and Bryan W. Berger ${ }^{1}$ \\ ${ }^{1 .}$ Department of Chemical and Biomolecular Engineering, Lehigh University, Bethlehem, PA 18015, \\ USA. \\ ${ }^{2}$ Department of Materials Science and Engineering, Lehigh University, Bethlehem, PA 18015, USA.
}

Quantum dots (QDs) are semiconductor nanocrystals that possess size-dependent photoluminescent properties due to quantum confinement effects. They have potential applications in many fields, including cancer cell tracking, LED lighting, and solar cells. Conventionally, QDs are producedin organic solvents via multi-step, batch processes at high temperatures and pressures, which results in limited production scale and high cost [1]. We have developed a novel, scalable approach for the biosynthesis of water-soluble CdS QDs using a strain of Stenotrophomonas maltophilia (SMCD1) to control particle size and hence QD photoluminescence through variation of growth time.Aberration-corrected scanning transmission electron microscopy (AC-STEM) and high-resolution electron microscopy (HREM) have provided us with valuable insight into the crystal structureand particle size of the biosynthesized CdS QDs.

The ability of prokaryotes like S. maltophilia to transform toxic levels of heavy metals (e.g.cadmium) into insoluble metal precipitates has inspired the pursuit of a range of biological approaches to synthesize QDs. S. maltophilia was isolated from soil and iteratively selected for variants in culture that were tolerant to cadmium acetate at concentrations in excess of $1 \mathrm{mmol}$ [2]. The selected cadmium-tolerant colonies were cultured in M9 minimal media containing L-cysteine and cadmium acetate. From the measured photoluminescence and absorption spectra, we confirmed the existence of fluorescent CdS QDs with a well-defined first excitonic peak. In addition, the reproducibility of the biosynthetic protocol was confirmed as different batches of growth culture under the same growth conditionsresulted in almost identical QD optical properties.

Photoluminescence was retained in the culture supernatants after removal of the cells by centrifugation, indicating extracellular production of CdS QDs. We also found that the optical properties of the QDs correlated with growth time in culture (Fig. 1). The peaks of the absorption spectra (Fig. 1(b)) and the fluorescence spectra (Fig. 1(c)) both shifted to higher wavelengths with increasing growth time. This trend impliesa gradual increase in the mean QD size with growth time. These results are most consistent with a sequential mechanism involving an initial rapid QD nucleation stage, followed by continuous particle growth in culture.

AC-STEM was utilized to identify the crystal structure of the biosynthesized CdS QDs, since X-ray and electron diffraction were unable to distinguish the two possible polymorphs of $\mathrm{CdS}$ (i.e. zinc-blende and wurtzite) due to the similar lattice spacings of the two structures and the nanoscopic nature of the QDs.Highlylocalized crystallographic information from individual particles could be obtained via high-angle annular dark field (HAADF) imaging in STEM mode, enabling us to measure the d-spacings and interplanar angles and compare them against the two CdS polymorphs. Fig. 2 shows examples of two CdS QDs whose lattice images match with the zinc-blende (Fig. 2(a)) and wurtzite (Fig. 2(b)) structures respectively. Thus we conclude that both zinc-blende and wurtzite structures co-exist for CdS QDs produced via strain SMCD1. To directlydetermine particle size distributions of the QDs, 
HREMimaging was used to preferentially pick up the periodicities in nanocrystals with well-defined particle edges. The mean particle size was found to increase with growth time, and could be correlated with the evolution of the optical spectra with increasing growth time [3].

\section{References:}

[1] K. Sanderson, Nature 459 (2009), p. 760.

[2] C. Bollet, A. Davin-Regli and P. De Micco, Appl. Environ. Microbiol. 61 (1995), p. 1653.

[3] The authors acknowledge funding from National Science Foundation.

a)

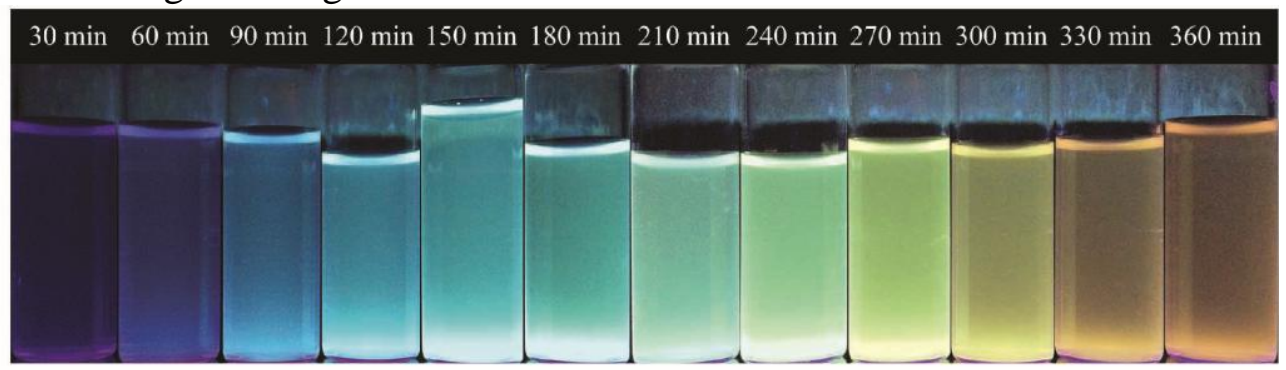

b)

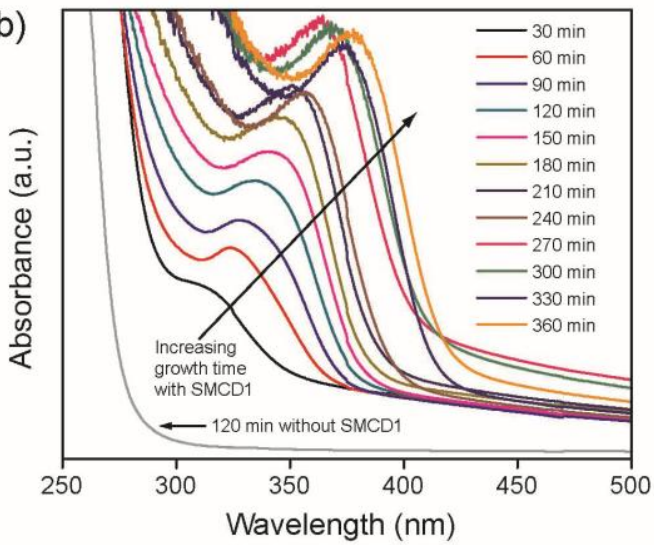

c)

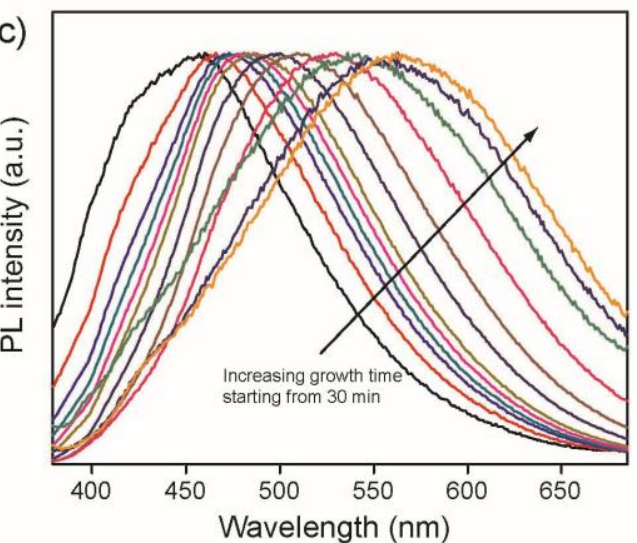

Figure 1.Optical properties of purified, biosynthetic CdS QDs of different sizes. (a) Photograph of the culture supernatants from strain SMCD1 collected at various growth times (30-360 min) when illuminated under UV light; (b) UV-vis absorption spectra of CdS QDs as a function of culture growth time; (c) Fluorescence emission spectra using $350 \mathrm{~nm}$ excitation of CdS QDs as a function of culture growth time.

a)

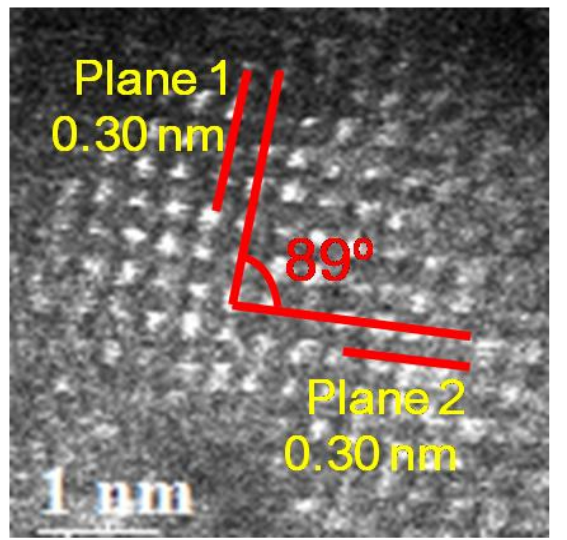

b)

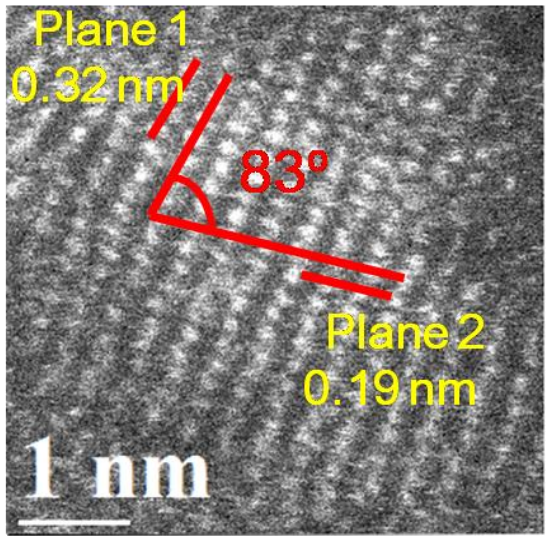

Figure 2.HAADF images of individual CdS particles after $60 \mathrm{~min}$ of growth exhibiting (a) zinc-blende and (b) wurtzite structures respectively. 